\title{
Septic arthritis in an unusual localization
}

\author{
Lia Marques*, André Simões, Sara Úria \\ Serviço de Medicina III, Hospital Pulido Valente, Centro Hospitalar Lisboa Norte, Lisboa, Portugal; \\ *Corresponding Author: marques.lia@gmail.com
}

Received 5 June 2013; revised 30 June 2013; accepted 5 July 2013

Copyright (C) 2013 Lia Marques et al. This is an open access article distributed under the Creative Commons Attribution License, which permits unrestricted use, distribution, and reproduction in any medium, provided the original work is properly cited.

\begin{abstract}
Septic arthritis is a rheumatological emergency due to its potential for rapid articular destruction and permanent functional loss. Its incidence ranges between 4 and 29 cases per 100,000 person-years, and depends on population variables and preexisting structural joint abnormalities. Clinical manifestations, severity, treatment and prognosis depend on the aetiologic agent, patient basal status and articulation involved. The sternoclavicular and condrosternal articulations are rarely affected. A $\mathbf{2 4}$ years old man presented with fever and right shoulder pain. Physical examination revealed swelling, redness, increased local heat, intense pain and functional impairment of the patient's right shoulder. Laboratory inflammatory markers were elevated. Right sternoclavicular articulation ultrasound, right sternoclavicular articulation X-ray, and galium bone scan have shown sternoclavicular arthritis and medial clavicular osteomyelitis. Blood cultures identified Staphylococcus aureus methicillin sensitive. The patient completed a six week antibiotic regimen and physical rehabilitation program. Herein, the authors report a case of sternoclavicular septic arthritis complicated with osteomyelitis and review aetiology, diagnosis, treatment and prognosis of this rare medical condition.
\end{abstract}

Keywords: Septic Arthritis; Sternoclavicular; Non-Gonococcal

\section{INTRODUCTION}

The incidence of septic arthritis ranges widely from 2 to $5 / 100,000 /$ year in the general population, 28 to $38 / 100,000 /$ year in patients with rheumatoid arthritis, and 40 to $68 / 100,000 /$ year in patients with joint prosthesis $[1,2]$. The most common route of articular infection is hematogenous spread during bacteremia [3,4]. Once in the joint, microorganisms are deposited in the synovial membrane, causing an acute inflammatory response. Inflammatory mediators and pressure from large effusions lead to the destruction of joint cartilage and bone loss $[3,5,6]$. The large joints are most commonly affected, and monoarticular infection is the rule [4,7]. Infections of axial joints, such as the sternoclavicular usually occur in patients with a history of intravenous drug abuse, immunossuppression, bacteremia or as a complication of a subclavian vein central venous catheter [3,8].

Gonococcal arthritis is the most common cause of acute monoarthritis in sexually active young adults [4]. Bacterial causes of septic arthritis include staphylococci (40\%), streptococci (28\%), gram-negative bacilli, mycobacteria, gram-negative cocci, gram-positive bacilli, and anaerobes [3,9]. S. aureus is the most common organism that causes nongonococcal arthritis. Some of the virulence factors of Staphylococcus aureus include collagenbinding protein, clumping factor $\mathrm{A}$ and $\mathrm{B}$, fibronectinbinding protein $\mathrm{A}$ and $\mathrm{B}$, capsular polysaccharide protein A, toxic shock syndrome toxin-1 and enterotoxins [10].

The classic presentation of non-gonococcal septic arthritis is the acute onset of pain, swelling, and decreased range of motion in a single joint. Most patients are febrile, but constitutional symptoms such as fever, chills or rigours have low sensitivities [3,11]. There is often underlying illnesses and predispositions to infections, such as intravenous drug abuse; presence of prosthetic joints; neoplasia, renal failure, and rheumatoid arthritis $[1,12]$. Physical examination reveals the warmth and tenderness of the affected joint, joint effusion, and limited active and passive range of motion [3].

Arthrocentesis and synovial fluid analysis should be performed for all patients who present to an inflamed joint. Synovial fluid should be evaluated for white blood cells (WBC) count with differential, crystal analysis, Gram stain, and culture [3,13,14]. For joints that are deeper and more difficult to aspirate, ultrasound-guided or fluoroscopy-guided needle aspiration should be done [4]. In 
synovial fluid, a WBC count of more than 50,000 per $\mathrm{mm}^{3}$ and a polymorphonuclear cell count greater than 90\% directly correlate with infectious arthritis, but this overlaps with crystalline disease [3,11,15]. In patients without previous antibiotic treatment, synovial fluid cultures are positive in $70 \%$ to $90 \%$ of nongonococcal bacterial arthritis [15]. Blood cultures are positive in $40 \%$ to $50 \%$ of cases and are the only method of identifying the pathogen in $10 \%$ of cases [3,15-17].

Although inflammatory markers such as erythrocyte sedimentation rate (ESR), C-reactive protein (CRP), and WBC, are usually raised, their sensitivity is low $[18,19]$. There are no data on imaging studies that are pathognomonic for acute septic arthritis. Ultrasonography is more sensitive for detecting effusions, particularly in difficult to examine joints, such as acromioclavicular, or sternoclavicular [3]. Computed tomography (CT) and magnetic resonance imaging (MRI) can provide useful images to delineate the extent of the infection $[4,20]$. Triple-phase bone scintigraphy is the preferred modality of investigation for multiple articular involvement [4].

A clinical suspicion of a joint infection warrants the immediate initiation of antibiotic therapy [4]. Delays in treatment increase morbidity and mortality. A consensual initial empiric therapy to cover gonococci, S. aureus, and streptococci is ceftriaxone plus vancomycin pending final culture results [4]. In healthy, young, sexually active individuals with community-acquired septic arthritis and a negative synovial fluid gram-stained smear, ceftriaxone is a reasonable option to cover $N$. gonorrhoeae. Narrow antibiotic coverage is indicated if gram-positive cocci are found in the synovial fluid, and there is a primary source of staphylococcal skin infection. Appropriate monotherapy in this case may be a penicillinase-resistant penicillin or vancomycin if methicillin resistance is likely [4]. During the first few days of management, immobilization of the infected joint by external splinting and adequate analgesic administration ensure patient comfort. Physical therapy should be instituted as soon as the patient can tolerate it because early active range-of-motion exercises are beneficial for ultimate functional recovery [4].

Before antibiotics were available, two-thirds of patients died from septic arthritis [3]. Current mortality rates of bacterial arthritis range from $10 \%$ to $20 \%$, depending on the presence of comorbid conditions, such as older age, coexisting renal or cardiac disease, and concurrent immunosuppression [3].

Even with all incremental knowledge of the pathogenesis of septic arthritis caused by the common organisms, such as Staphylococcus aureus, treatment and outcome, of septic arthritis, have not improved substantially over the past 30 years $[4,21]$ making it important to notify and discuss clinical cases of septic arthritis, particu- larly those with special context such as the case presented here.

\section{CASE REPORT}

A 24 years old male, single, professional volleyball player, with no previous clinical history was observed at the emergency room for a 4-days story of fever (axillary temperature: $39.2^{\circ} \mathrm{C}$ ) with chills, and a fever free period of 6 hours after acetaminophen ingestion. He had also a 3-weeks pain localized in his right infraclavicular region, irradiating to the right shoulder, without a previous trauma. There were no other symptoms associated. He was monogamist, maintained unprotected sexual intercourse, and had no risk factors for particular infectious diseases. One month previous to hospital admission he had an incise skin wound in the right dorsal region, at the time without any disinfection. For the right infraclavicular pain he had already seek medical attention being diagnosed tendinitis and prescribed nonsteroidal anti-inflammatory drugs and mesotherapy.

Physical examination revealed: dehydration, fever (tympanic temperature $39.5^{\circ} \mathrm{C}$ ), normal blood pressure and sinus tachycardia (heart rate $110 \mathrm{bpm}$ ), swelling, redness, increased local heat, intense pain and functional impairment (abduction and adution) in his right shoulder. The investigations yielded the following results: white blood

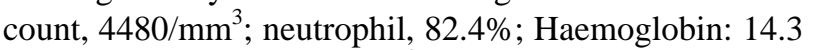
g/dL, platelets: $240,000 / \mathrm{mm}^{3}$. Blood C-reactive protein: $9.5 \mathrm{mg} / \mathrm{L}$; Creatinine $1.2 \mathrm{mg} / \mathrm{dL}$; Urea $23 \mathrm{mg} / \mathrm{dL}$; Chest $\mathrm{X}$-ray, right shoulder X-ray, clavicle and sternoclavicular join X-ray, right shoulder ultrasound and right shoulder computerized tomography (CT) has shown no structural abnormalities.

The patient was admitted to an internal medicine ward where investigation for febrile illnesses revealed: viral serological investigations were negative; transthoracic echocardiogram without abnormalities or valvular vegetations. For the first 48 hours the patient remained febrile and there were worsening inflammatory signs in right infraclavicular region, he was then admitted with a presumptive diagnosis of septic arthritis of the right sternoclavicular articulation. He was started with IV Ceftriaxone. At fourth antibiotic treatment day the patient had sustained apyrexia with reduction of laboratory inflammation markers. Blood cultures yielded Staphylococcus aureus methicillin sensitive (in five separate blood samples). Antibiotic regimen was changed to oxacillin according to sensibility testing. The patient was submitted to right clavicular ultrasound that has shown: liquid image in the sternoclavicular articulation measuring $39 \times$ $24 \times 0.5 \mathrm{~mm}$ with calcification representing arthritis (Figure 1). A right sternclavicular articulation X-ray was performed: inferior medial clavicular erosion (Figure 2). A galium bone scan has shown osteomielitis of the me- 


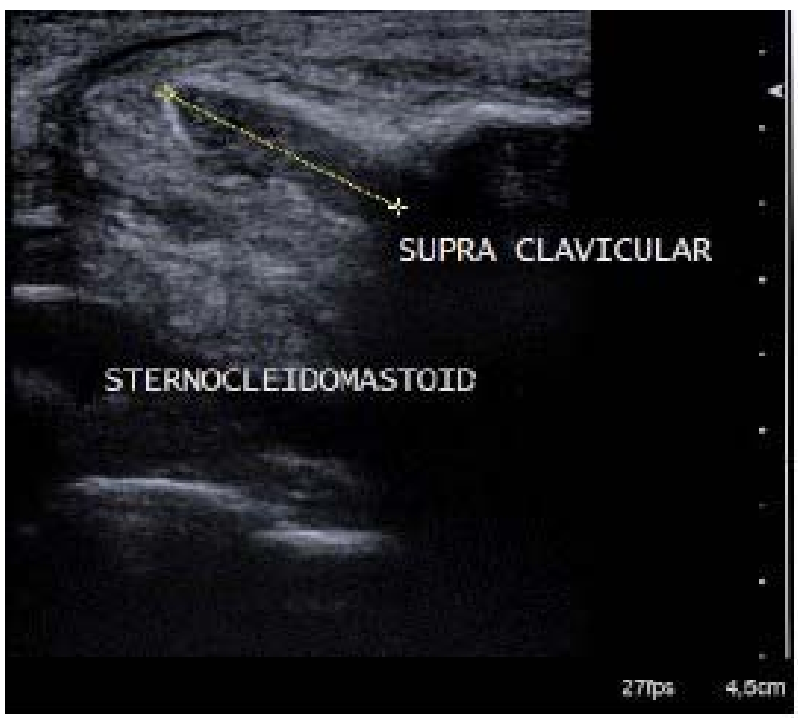

Figure 1. Right clavicular ultrasound showing liquid image in the sternoclavicular articulation measuring $39 \times 24 \times 0.5 \mathrm{~mm}$ with calcification representing arthritis.

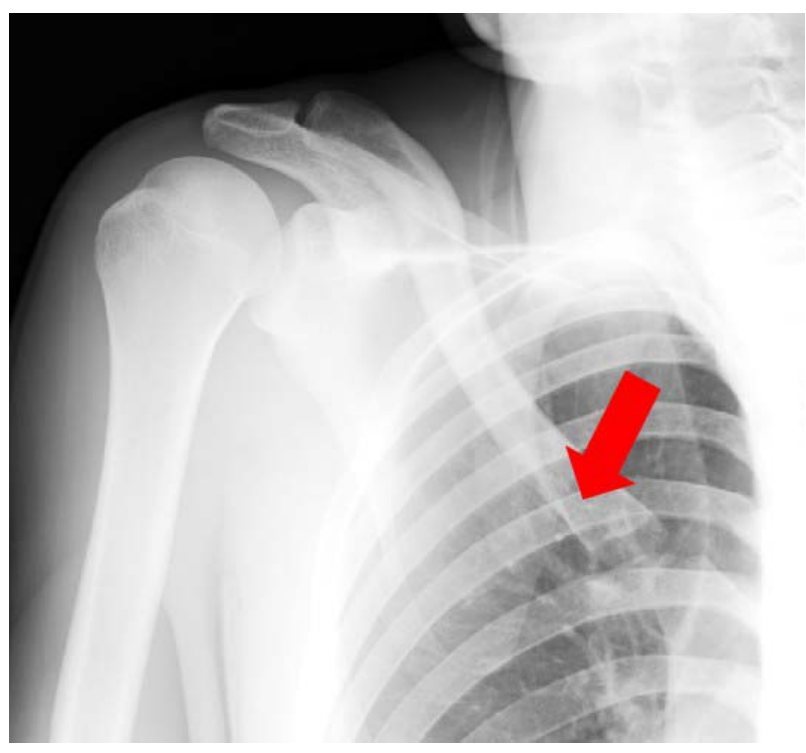

Figure 2. A right sternclavicular articulation X-ray with inferior medial clavicular erosion.

dial half of right clavicle (Figure 3). The patient completed a 14 days regimen of parenteral antibiotic therapy with oxacillin 2 gr every 6 hours. Followed by oral fluoxacillin for another 6 weeks. One year of intense physical therapy he came back to his active professional life as a volleyball player.

\section{DISCUSSION}

Septic arthritis incidence appears to be increasing, probably due to orthopedic procedures, an aging population, and the increased use of immunosupressive therapy [15]. Even so, the sternoclavicular articulation is rarely

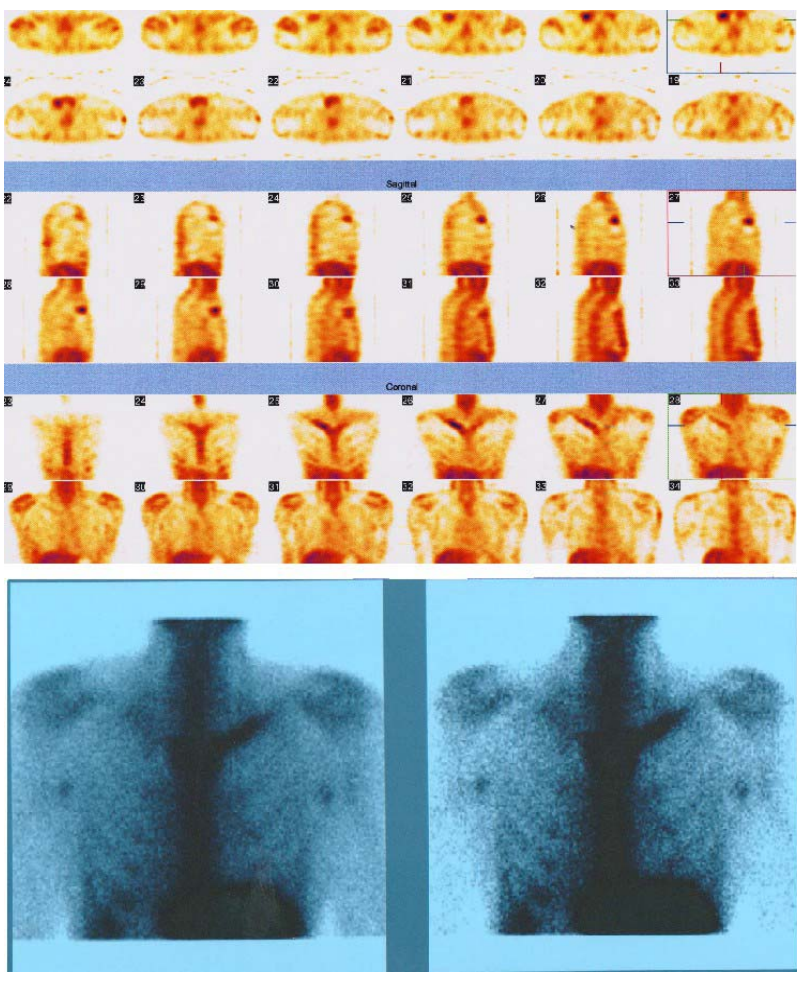

Figure 3. Galium bone scan showing osteomielitis of the medial half of right clavicle.

affected, with an incidence of 5\% [4,22]. A case of septic arthritis of the right ster oclavicular articulation complicated with osteomyeliltis is reported. There are no classical risk factors identified for septic arthritis in this patient, but previous articular damage due to the patient profession may represent a susceptibility factor. A main source of hematogenous spread to septic arthritis is skin lesions, as in this case [3]. The definitive diagnosis of septic arthritis is established through the isolation of the bacteria in the articular fluid [4]. In the reported case arthrocentesis was attempted but wasn't possible due to lack of ultrasound window for the exam. Even so, the clinical setting associated with the positive blood cultures and imagiologic findings are representative of septic arthritis. Usually plain films are initially normal, but often show nonspecific changes of inflammatory arthritis, and may reveal complicating osteomyellitis [4]. When the identity and the sensitivities of the organism are known, antibiotic therapy should continue with the most efficacious agent that has the best safety profile and narrowest spectrum. The parenteral route of antibiotic administration is the preferred initial treatment [4]. The optimal duration of antibiotic treatment has not been prospectively studied. For non-gonococal septic arthritis therapy ranging from 2 weeks to 6 weeks is recommended. Parenteral antibiotics may be switched to oral antibiotics after 2 weeks provided that there is clinical improvement, inflammatory markers are trending down, and oral anti- 
biotics are available to which the microorganism is susceptible [4,17]. Staphylococcal septic arthritis usually requires 3 to 4 weeks of therapy $[6,23]$. Due to the presence of osteomyelitis antibiotic therapy was prolonged to 6 weeks in this patient.

The outcome of treated septic arthritis can be measured as mortality and functional outcome of the infected joint. After completing antimicrobial therapy, patients with $S$. aureus septic arthritis regain $46 \%$ to $50 \%$ of their baseline joint function [3,16]. Morbidity (e.g., amputation, arthrodesis, prosthetic surgery, severe functional deterioration) occurs in one-third of patients with bacterial arthritis, usually affecting older patients, those with preexisting joint disease, and those with synthetic intraarticular material [3]. This patient had a long term full functional articular recovery.

This case report highlights that septic arthritis can be particularly difficult to diagnose and treat if it occurs in fibrocartilaginous joints such as the sternoclavicular joint. It also reinforces the need to consider the diagnosis of septic arthritis in any patient with acute inflammatory arthritis, since prompt diagnosis and treatment of infectious arthritis can prevent significant morbidity and mortality.

\section{REFERENCES}

[1] Kaandorp, C.J., Van Schaardenburg, D., Krijnen, P., et al. (1995) Risk factors for septic arthritis in patients with joint disease: A prospective study. Arthritis \& Rheumatism, 38, 1819-1825. doi:10.1002/art.1780381215

[2] Geirsson, A.J., Statkevicius, S. and Vikingsson, A. (2008) Septic arthritis in Iceland 1990-2002: Increasing incidence due to iatrogenic infections. Annals of the Rheumatic Diseases, 67, 638-643.

doi:10.1136/ard.2007.077131

[3] Horowitz, D.L., Katzap, E., Horowitz, S. and BarillaLaBarca, M.-L. (2011) Approach to septic arthritis. American Family Physician, 84, 653-660.

[4] Cook, P.P. and Siraj, D.S. (2012) Bacterial arthritis. Firestein: Kelley's textbook of rheumatology. 9th Edition, Elsevier, Saunders, Philadelphia.

[5] Goldenberg, D.L. (1998) Septic arthritis. Lancet, 351, 197202. doi:10.1016/S0140-6736(97)09522-6

[6] Smith, J.W. and Piercy, E.A. (1995) Infectious arthritis. Clinical Infectious Diseases, 20, 225-230. doi:10.1093/clinids/20.2.225

[7] Epstein, J.H., Zimmermann III, B. and Ho Jr., G. (1986) Polyarticular septic arthritis. Journal of Rheumatology, 13, 1105-1107.

[8] Ross, J.J. and Shamsuddin, H. (2004) Sternoclavicular septic arthritis: Review of 180 cases. Medicine (Baltimore), 83, 139-148.

[9] Ryan, M.J., Kavanagh, R., Wall, P.G. and Hazleman, B.L. (1997) Bacterial joint infections in England and Wales:
Analysis of bacterial isolates over a four year period. British Journal of Rheumatology, 36, 370-373. doi:10.1093/rheumatology/36.3.370

[10] Patti, J.M., Allen, B.L., McGavin, M.J., et al. (1994) MSCRAMM-mediated adherence of microorganisms to host tissues. Annual Review of Microbiology, 48, 585-617. doi:10.1146/annurev.mi.48.100194.003101

[11] Margaretten, M.E., Kohlwes, J., Moore, D. and Bent, S. (2007) Does this adult patient have septic arthritis? JAMA, 297, 1478-1488. doi:10.1001/jama.297.13.1478

[12] Mathews, C.J., Weston, V.C., Jones, A., et al. (2010) Bacterial septic arthritis in adults. Lancet, 375, 846-855. doi:10.1016/S0140-6736(09)61595-6

[13] Schumacher Jr., H.R. (2001) Synovial fluid analysis and synovial biopsy. In Ruddy, S., Harris, E.D., Sledge, C.B. and Kelley, W.N., Eds., Kelley's Textbook of Rheumatology, 6th Edition, Saunders, Philadelphia.

[14] Shmerling, R.H., Delbanco, T.L., Tosteson, A.N. and Trentham, D.E. (1990) Synovial fluid tests. What should be ordered? JAMA, 264, 1009-1014. doi:10.1001/jama.1990.03450080095039

[15] Mathews, C.J., Weston, V.C., Jones, A., Field, M. and Coakley, G. (2010) Bacterial septic arthritis in adults. Lancet, 375, 846-855. doi:10.1016/S0140-6736(09)61595-6

[16] Weston, V.C., Jones, A.C., Bradbury, N., et al. (1999) Clinical features and outcome of septic arthritis in a single UK health district 1982-1991. Annals of the Rheumatic Diseases, 58, 214-219. doi:10.1136/ard.58.4.214

[17] Coakley, G., Mathews, C., Field, M., et al. (On Behalf of the British Society for Rheumatology Standards) (2006) Guidelines and Audit Working Group: BSR \& BHPR, BOA, RCGP and BSAC guidelines for management of the hot swollen joint in adults. Rheumatology (Oxford), 45, 1039-1041. doi:10.1093/rheumatology/kel163a

[18] Li, S.F., Henderson, J., Dickman, E., et al. (2004) Laboratory tests in adults with monoarticular arthritis: Can they rule out a septic joint? Academic Emergency Medicine, 11, 276-280. doi:10.1111/j.1553-2712.2004.tb02209.x

[19] Gupta, M.N., Sturrock, R.D. and Field, M. (2001) A prospective 2-year study of 75 patients with adultonset septic arthritis. Rheumatology (Oxford), 40, 24-30. doi:10.1093/rheumatology/40.1.24

[20] Sanchez, R.B. and Quinn, S.F. (1989) MRI of inflammatory synovial processes. Magnetic Resonance Imaging, 7, 529-540. doi:10.1016/0730-725X(89)90406-2

[21] Goldenberg, D.L. (1994) Bacterial arthritis. Current Opinion in Rheumatology, 6, 394-400. doi:10.1097/00002281-199407000-00008

[22] Kaandorp, C.J.E., Dinant, H.J., van de Laar, M.A.F.J., et al. (1997) Incidence and sources of native and prosthetic joint infection: A community based prospective survey. Annals of the Rheumatic Diseases, 56, 470-475. doi:10.1136/ard.56.8.470

[23] Ross, J.J., Saltzman, C.L., Carling, P., et al (2003) Pneumococcal septic arthritis: Review of 190 Cases. Clinical Infectious Diseases, 36, 319-327. doi:10.1086/345954 